\title{
Fatigue phenomena in thin ferroelectric films stimulated by repeated switching of the polarization
}

\author{
A. S. Sidorkin**, P. Saint-Gregoire ${ }^{\dagger}$ and L. P. Nesterenko* \\ *Experimental Physics Department, Voronezh State University \\ University Sq., 1, Voronezh, 394006, Russia \\ ${ }^{\dagger}$ MIPA Laboratory, University of Nimes \\ Department of Sciences and Arts, Pl Gabriel Peri \\ 30021 Nimes Cedex 01, France \\ and \\ ICGM (UMR CNRS Nr 5253) \\ C2M, UM2, 34095 Montpellier cedex, France \\ tsidorkin@phys.vsu.ru
}

Received 25 September 2012; Revised 22 November 2012; Accepted 4 January 2013; Published 11 March 2013

\begin{abstract}
With the use of techniques of Sawyer-Tower and Mertz method regularities of the origin of "fatigue" in thin ferroelectric films of lead titanate and lead zirconate titanate have been investigated in the fields above coercive. We propose a model to explain the experimentally observed switching-dependent fatigue processes by the motion of charged dislocations in an applied external alternating electric field.
\end{abstract}

Keywords: Ferroelectric; switching; domain boundary; fatigue; hysteresis; coercive field.

\section{Introduction}

Most practical applications of ferroelectrics are related to the change of the polar state. One of the promising devices of modern nanoelectronics is the nonvolatile memory with high capacity, which is based on recording of nanodomain pictures in ferroelectric films using the probe technique. ${ }^{1-3}$ The wide commercialization of such devices is limited by a number of problems including the so-called effect of fatigue, which consists in decreasing the switched polarization with an increase in the number of switchings by an external electric field.

To explain the observed processes a few basic types of models of fatigue were proposed. One of the earliest are the models in which the reduction of switchable polarization is associated with a decrease in the area of the electrodes as a result of burning or peeling, and also with the development of mechanical stresses in the region near the electrode as a result of multiple cycling. ${ }^{4-6}$ In the other type of models the fatigue phenomenon is attributed to the blocking of switching processes by crystal lattice defects in the bulk of material, and on its surface..$^{7-10}$ Near-surface blocking of domain processes can be achieved by charge injection from the electrodes, ${ }^{1-13}$ or due to electromigration to the surface of already existing material defects such as oxygen vacancies in the oxygen-containing films with perovskite structure. ${ }^{14,15}$

A special mechanism of fatigue is the development during repeated switching of the surface non-ferroelectric layer, which leads to a redistribution of the electric field in the formed heterostructure and, consequently, to change the field acting in ferroelectric. ${ }^{16-18}$
General regularities of the fatigue process as a dependence on the amplitude of the field, stimulating switch, its frequency and temperature, can be formally explained in several models directly. In these circumstances, to identify the benefits of any particular model seems expedient to conduct specially designed experiments to detect signs reflecting the individual characteristics of a particular model.

Our preliminary studies show that the observed fatigue processes have a domain-related nature, at least in the case of films of lead titanate and lead zirconate titanate. ${ }^{19,20}$ It is also clear that in these processes the domain structure can play both an active or a passive role, when in the first case, the basic parameters of the switching process change and in the other it is simply blocked by extraneous factors. In this context, the purpose of the present work was the experimental study of the fatigue processes in lead titanate and lead zirconate titanate ferroelectric thin films with a view to reveal the specific role of the domain structure in the phenomena under consideration.

\section{Sample Preparation and Structural Investigations}

\subsection{Synthesis of lead titanate thin films by magnetron sputtering}

In the present work, the investigation of fatigue processes was carried out on films prepared by two methods: magnetron sputtering and sol-gel method. Under the method of magnetron sputtering lead titanate $\mathrm{PbTiO}_{3}$ thin films were prepared using layer-by-layer deposition of titanium and lead 
onto substrates. VK-100-1 polycrystalline polikor $\left(\mathrm{Al}_{2} \mathrm{O}_{3}\right)$ and $\mathrm{Si}(100)$ single crystal silicon were used as substrates. Metal layers were deposited onto unheated substrates in a plasma-forming argon medium. The sputtering was performed in a single experimental cycle, and the chamber was preliminarily evacuated to a pressure of $0.33 \times 10^{-2} \mathrm{~Pa}^{21}$ The thickness of each layer ( $\mathrm{Pb}$ or $\mathrm{Ti}$ ) set the time of the process at a known average sputtering rate. The average sputtering rate of each component was determined experimentally. The sputtering time ratio and, consequently, the thickness ratio for lead and titanium was chosen according to the stoichiometry of $\mathrm{PbTiO}_{3}$. The total thickness of metal layers was chosen corresponding to the required thickness of the film.

In order to compensate for lead evaporated in the process of film deposition, the ratio $\mathrm{Pb} / \mathrm{Ti}$ between the mole fractions of the initial components was chosen experimentally (varying from 1.05 to 1.30 ). Polycrystalline films with different ratios between the $\mathrm{Ti}$ and $\mathrm{Pb}$ components were prepared by varying the sputtering time of metals at a constant sputtering rate. The structure and composition of the films were determined using the $\mathrm{X}$-ray analysis ( $\mathrm{Cu} \mathrm{K} \alpha$ radiation). It was experimentally established that the films with the ratio between lead and titanium in the initial components $\mathrm{Pb} / \mathrm{Ti}=1.25$ have a composition most similar to the stoichiometric one and exhibit the most pronounced ferroelectric properties. In this case, the X-ray diffraction patterns contain only the reflections corresponding to the $\mathrm{PbTiO}_{3}$ perovskite phase.

The high-temperature annealing of sputtered structures was performed in resistance heating furnace in an oxygen atmosphere. The annealing conditions for the prepared layered structures were varied depending on the results of the X-ray analysis and investigation of the ferroelectric properties. The experimental investigations have revealed that the optimum annealing conditions are the gradual heating of the sample from room temperature up to temperatures of $700-750^{\circ} \mathrm{C}$ for one hour and slowly cooling (within $3-4 \mathrm{~h}$ ) to room temperature. According to the X-ray analysis data, the $\mathrm{PbTiO}_{3}$ phase in the synthesized samples is formed only at temperatures above $650^{\circ} \mathrm{C}$. Annealing at a temperature of $650^{\circ} \mathrm{C}$ appeared to be insufficient for the complete disappearance of unreacted oxides. Prolonged annealing at a temperature of $750^{\circ} \mathrm{C}$ resulted in an increase in the content of the nonstoichiometric phases $\mathrm{PbTi}_{3} \mathrm{O}_{7}$. The final film thickness after annealing varied from 0.1 to $1.0 \mu \mathrm{m}$.

The important annealing stage is the gradual (for 3-4h) cooling of the sample, which significantly affects the shape and the size of crystallites. Investigation of surface microstructure of the obtained films using an atomic force microscope Femtoscan-001-Online, and Scanning Electron Microscope JEOL JSM-6380LV showed that the slow cooling leads to the intergrowth of crystallites throughout the film thickness: in the study of the edge of the deposited structure the columnar structure of grains is clearly distinguished in the synthesized films. Thus, using the method of magnetron sputtering we have obtained polycrystalline films with a sufficiently large size of grains (crystallites) - from 400 to $600 \mathrm{~nm}$ across width and from 600 to $900 \mathrm{~nm}$ across height.

\subsection{Preparation of lead zirconate titanate and lead titanate films by the sol-gel method}

Lead zirconate titanate films were synthesized by the sol-gel method from solutions with the component ratio $\mathrm{Pb}: \mathrm{Ti}: \mathrm{Zr}=1.25: 0.50: 0.50$. When preparing the initial reactants, lead, titanium, and zirconium were introduced in the form of lead acetate $\mathrm{Pb}\left(\mathrm{CH}_{3} \mathrm{COO}\right)_{2} \cdot 3 \mathrm{H}_{2} \mathrm{O}$, titanium - in the form of n-butoxide $\mathrm{Ti}\left(\mathrm{OC}_{4} \mathrm{H}_{9}\right)_{4}$, and zirconium - in the form of nitrate $\mathrm{Zr}\left(\mathrm{NO}_{3}\right)_{4} \cdot 5 \mathrm{H}_{2} \mathrm{O}$, respectively. 2-Methoxyethanol $\mathrm{HOCH}_{2} \mathrm{CH}_{2} \mathrm{OCH}_{3}$ as a solvent and acetylacetone $\mathrm{CH}_{3}$ $\mathrm{COCH}_{2} \mathrm{COCH}_{3}$ as a chemical modifier were added for the preparation of the working solution. The prepared solution was homogeneous, transparent, and stable under normal conditions and possessed a good wettability with respect to the substrate. The lead acetate and zirconium nitrate were initially dissolved in 2-methoxyethanol and distilled (dried) at a temperature of $100^{\circ} \mathrm{C}$ for approximately $1 \mathrm{~h}$. After distillation, the required amount of acetylacetone was added in order to stabilize the solution. Final drying was performed at a temperature of $125^{\circ} \mathrm{C}$ for the same time. After cooling to room temperature, the required amount of titanium $n$-butoxide was added to the solution stirred at a temperature of $80^{\circ} \mathrm{C}$. The prepared solution can be stored in an argon inert atmosphere for several months with retention of the necessary properties without crystallization. The whole process of preparing the working solution was carried out in air. The molar ratio $\mathrm{Zr}$ : $\mathrm{Ti}=0.5: 0.5$ was chosen, because this ratio is in the immediate vicinity to morphotropic boundary $(0.52 / 0.47)$ where the given material exhibits the most pronounced ferroelectric properties. Then, individual layers were deposited onto the polikor substrates spinning at a rate of 4000-5000 rpm and dried at a temperature of $180^{\circ} \mathrm{C}$ for $5 \mathrm{~min}$. The thickness of each layer was determined using the electron microscope and varied from 80 to $100 \mathrm{~nm}$. The films with different thicknesses could be prepared by varying the number of layers.

The chemical reaction of the formation of the perovskite phase (PZT) proceeded on the substrate in the final hightemperature annealing, which was performed in a resistance furnace in an oxygen atmosphere. Prepared samples were annealed in a temperature range from 450 to $750^{\circ} \mathrm{C}$, and subsequently the phase composition of these samples was studied. The phase composition of the films prepared by sol-gel technology was determined using a DRON diffractometer ( $\mathrm{CuK} \alpha$ radiation), as well as in the case of films prepared by magnetron sputtering. The analysis of the X-ray diffraction patterns showed that the $\mathrm{Pb}\left(\mathrm{Zr}_{0.5} \mathrm{Ti}_{0.5}\right) \mathrm{O}_{3}$ films annealed at a temperature of $450{ }^{\circ} \mathrm{C}$, for the most part, have an amorphous structure. The films annealed at a temperature of $500^{\circ} \mathrm{C}$ predominantly contain the pyrochlore phase. It is with an annealing temperature above $550^{\circ} \mathrm{C}$ that the perovskite phase grows and the pyrochlore phase progressively 


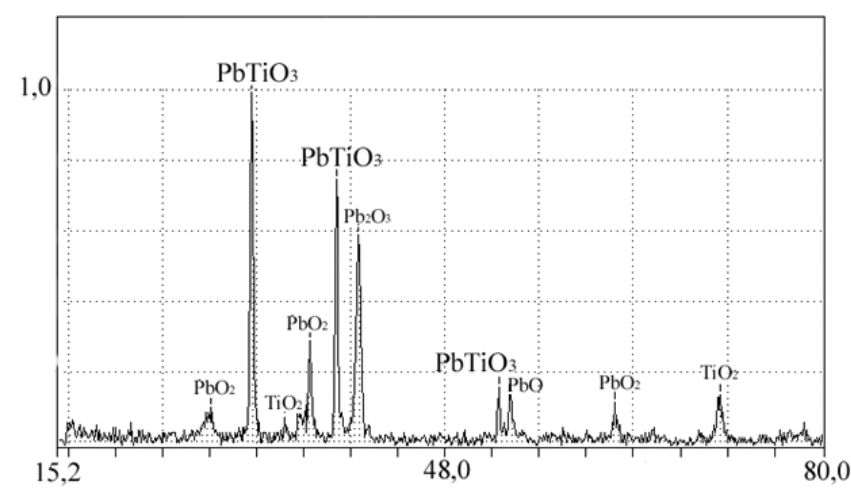

(a)

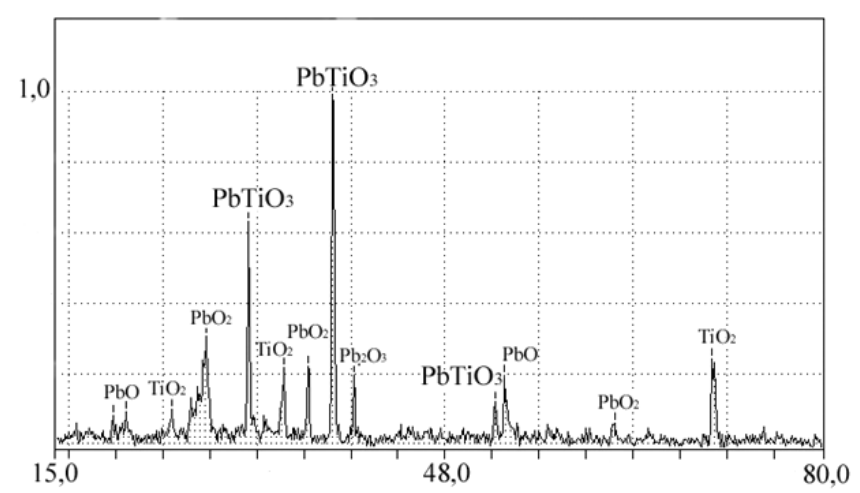

(b)

Fig. 1. Diffraction of lead titanate films with thickness of $500 \mathrm{~nm}$, obtained at different annealing conditions: (a) annealing temperature $700^{\circ} \mathrm{C}$ and (b) annealing temperature is $750^{\circ} \mathrm{C}$.

disappears. The perovskite phase becomes dominant at an annealing temperature of $600^{\circ} \mathrm{C}$. Annealing at temperatures above $650^{\circ} \mathrm{C}$ (i.e., at $700^{\circ} \mathrm{C}$ and $750^{\circ} \mathrm{C}$ ) leads to a deterioration of the phase composition. Thus, the results of XRD showed after annealing at $600-650^{\circ} \mathrm{C}$ all the samples prepared using sol-gel technology have a perovskite structure without any other phases admixtures. The crystallite sizes in these PZT films were equal to $100 \div 200 \mathrm{~nm}^{22}$

The stock solution for the synthesis of $\mathrm{PbTiO}_{3}$ films was prepared in a similar way but without adding the zirconium $n$-butoxide. The ratio between the components was $\mathrm{Pb}: \mathrm{Ti}=1.25: 1.00$. Subsequent deposition and drying of layers were carried out in the same manner as for the lead zirconate titanate films and at the same temperatures. For $\mathrm{PbTiO}_{3}$ films high-temperature annealing was performed at temperatures of $700-750^{\circ} \mathrm{C}$.

Figure 1 shows the diffraction patterns of lead titanate films annealed at different temperatures. It is seen that the increase of annealing temperature results in the increase of the intensity peak located around $39^{\circ}$ which is characteristic of the perovskite structure, and indicates the appearance of the chosen orientation of crystallites with indices (111). One can also note a decrease of the intensity of nonvolatile lead
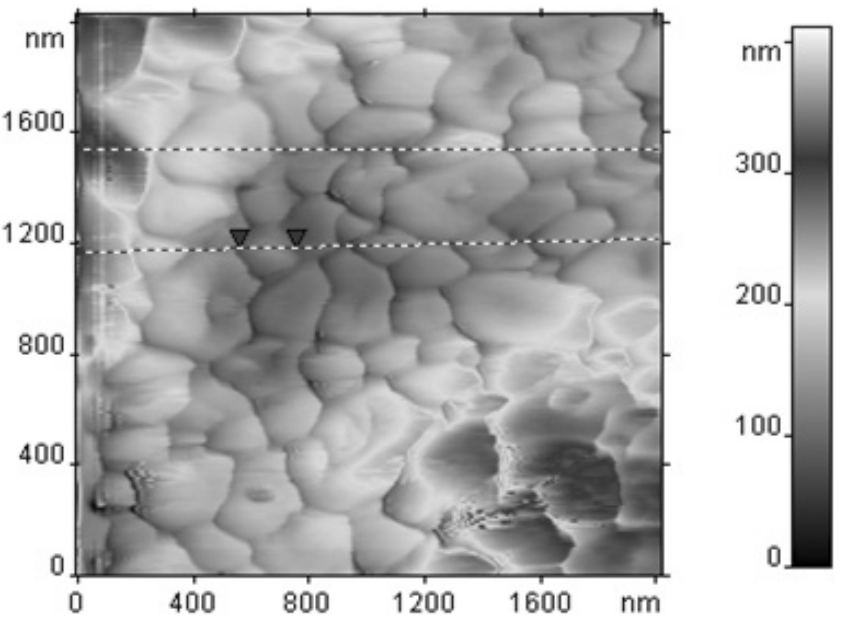

(a)
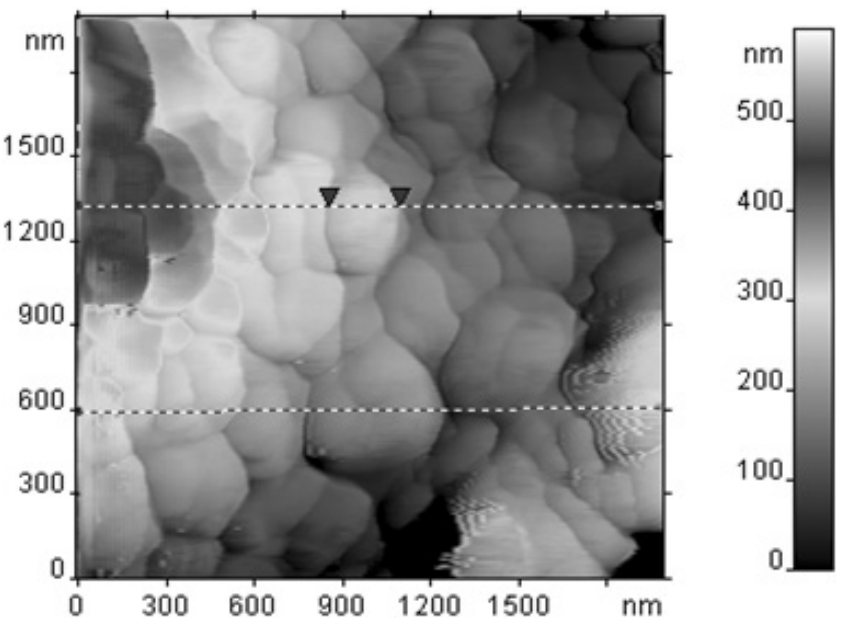

(b)

Fig. 2. AFM image of lead titanate film on silicon substrate at annealing temperature: (a) $700^{\circ} \mathrm{C}$ and (b) $750^{\circ} \mathrm{C}$.

oxide $\mathrm{Pb}_{2} \mathrm{O}_{3}$ which remains in the structure and can aggravate stoichiometry of the film.

Figure 2 shows the AFM-images of the surface and topography for the lead titanate films $\mathrm{PbTiO}_{3}$ with thickness of $500 \mathrm{~nm}$ which were synthesized on silicon substrates and were annealed in an oxygen atmosphere at $700^{\circ} \mathrm{C}$ and $750^{\circ} \mathrm{C}$, respectively. These figures clearly show the enlargement of the crystallite size with increasing annealing temperature of $200 \div 250 \mathrm{~nm}$ up to $300 \div 400 \mathrm{~nm}$.

\section{Techniques for Dielectric and Polarization Switching Measurements}

To define the repolarization properties and characteristics of the films two techniques have been used. The determination of reorientational (residual) polarization $P_{s}$, coercive field $E_{c}$ and the internal bias field $E_{b}$ was carried out by measuring the 
parameters of the dielectric hysteresis loops using a modified Sawyer-Tower circuit with the conductivity compensation.

Simultaneously, these parameters and characteristics related with the kinetics of polarization switching were measured using Mertz method. The sample was switched here using a rectangular voltage with a value from 2 to $8 \mathrm{~V}$ at a frequency of $1 \mathrm{kHz}$ to $1 \mathrm{MHz}$, which was applied to the ferroelectric capacitor with a film connected in series with the reference resistance. The external field strength was of $100 \div$ $800 \mathrm{kV} \cdot \mathrm{cm}^{-1}$ that was higher than the value of the coercive field $E_{c}$ in investigated films, whose value is determined in advance by the dielectric hysteresis loops.

The switching current was measured from the voltage drop across a series $50 \Omega$ resistance and was registered on the screen of a two-channel digital oscilloscope. The rise time of the switching voltage was less than $10 \mathrm{~ns}$. The measured quantity was the integral characteristics of the switching such as switching current pulse maximum $i_{\max }$ and total switching time $\tau_{s}$. The switching time $\tau_{s}$ was defined as the interval between the beginning of the pulse and the moment when the current drops to $10 \%$ of the maximum value $i_{\max }$.

In order to determine the temperature dependence of the dielectric permittivity and to study the dispersion of the permittivity of the synthesized films, the impedance of the samples was measured on a Solartron-1260 impedance analyzer with a dielectric interface in the frequency range from $1 \mathrm{kHz}$ to $30 \mathrm{MHz}$ and the temperature range from 20 to $650{ }^{\circ} \mathrm{C}$, including the temperatures of the ferroelectric and paraelectric phases. The measuring voltage was equal to $100 \mathrm{mV}$. The relative error in these measurements did not exceed $0.1 \%$.

Two types of samples were used for experimental studies. Samples of the first type are on polikor substrate with an applied system of platinum electrodes at the top and a resistive heating element at the lower surface (the substrate was soldered with gold wires into a standard chip). The second type of the samples was prepared on substrates of single crystal silicon with platinum Pt sublayer as a low electrode with thickness less than $500 \mathrm{~nm}$. Upper gold Au electrodes were plotted by thermal evaporation through a mask here for further electrical measurements.

\section{Investigation of the Fatigue of the Lead Titanate and Lead Zirconate Titanate Thin Films}

The results of control tests of the samples showed the presence in them of the first-order ferroelectric phase transition at a temperature of $\approx 495^{\circ} \mathrm{C}$ for lead titanate and at a temperature of $\approx 330^{\circ} \mathrm{C}$ for lead zirconate titanate, respectively, as evidenced by the hysteresis in the temperature dependence of the dielectric constant and abrupt decrease of the spontaneous polarization and coercive field to zero at $T_{c}$.

Fatigue associated with switching is characterized by two factors: causal (stimulant) is usually a variable electric field, and consequence is the changing polarization. In principle,

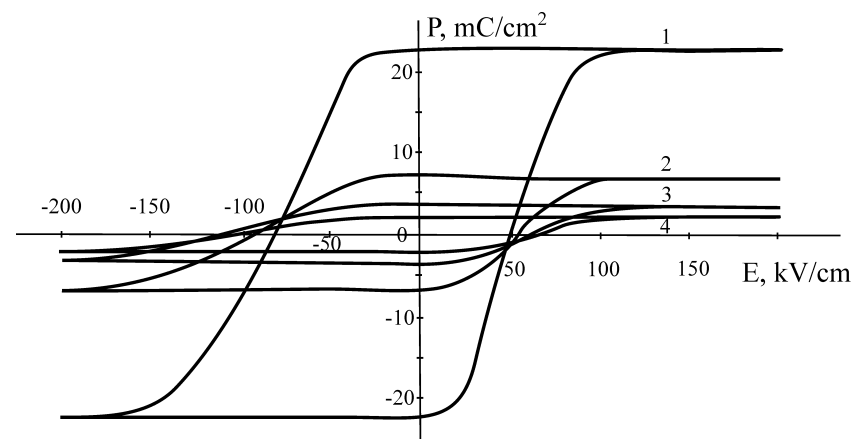

Fig. 3. Changing the hysteresis loops in an external field of $200 \mathrm{kV} /$ cm for $\mathrm{PbTiO}_{3}$ films: $1-N=0,2-N=3 \cdot 10^{9}, 3-N=6 \cdot 10^{9}$, $4-N=2.4 \cdot 10^{10}, 5-N=1.08 \cdot 10^{11}$.

each of them can be cause of fatigue phenomena detected in the experiment. In order to separate their effects in this paper we propose and implement an experiment to study the fatigue phenomenon in the electric fields with an amplitude greater than coercive, corresponding to the so-called "whiskers" of the hysteresis loop (Fig. 3). Obviously, changes of the applied field in this region should not affect the switching processes, which require the same coercive field, but at the same time, should affect the intensity of the charge drift. Taking into account that there are parts of the hysteresis loop, where the polarization reversal is only partially (from $25 \mathrm{kV} / \mathrm{cm}$ up to $100 \mathrm{kV} / \mathrm{cm}$ in Fig. 3), for the implemented experiment we selected those areas of loops where the polarization goes to full saturation (i.e., the applied field is greater than $100 \mathrm{kV} / \mathrm{cm}$ ).

The experimental results are illustrated in Figs. 4-7, which show that increasing the amplitude of the alternating electric field in the range of values above the coercive one leads to an acceleration of fatigue processes (i.e., to reduce the threshold of switching cycles in which the fatigue changes in the switching polarization and coercive field are observed). Simultaneously, the threshold of switching cycles increases with increasing frequency (Figs. 8 and 9).

Figure 5 shows the dependences of the switched polarization on the logarithm of the number of switching cycles for

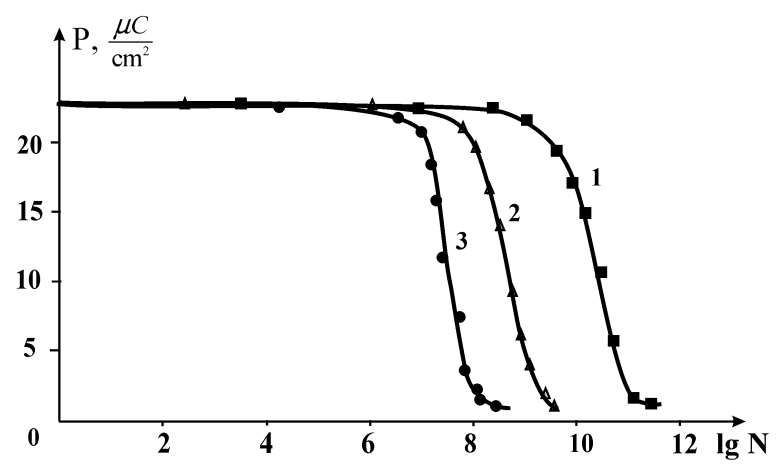

Fig. 4. Dependence of switched polarization on the number of switching cycles for $\mathrm{PbTiO}_{3}$ films on silicon substrate at $=200^{\circ} \mathrm{C}$, $f=10 \mathrm{MHz}: 1,2,3-E=130,200,270 \mathrm{kV} / \mathrm{cm}$. 


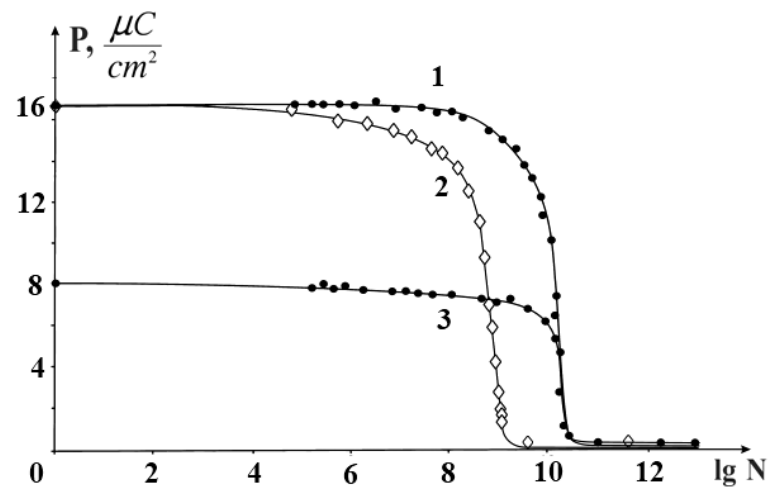

Fig. 5. Dependence of polarization of $\mathrm{PbTiO}_{3}$ films on the number of switching cycles at various temperatures and values of external applied field: $1-\mathrm{T}=480^{\circ} \mathrm{C}, E=70 \mathrm{kV} / \mathrm{cm} ; 2-480^{\circ} \mathrm{C}, 140 \mathrm{kV} /$ $\mathrm{cm} ; 3-490^{\circ} \mathrm{C}, 70 \mathrm{kV} / \mathrm{cm}$.

the $\mathrm{PbTiO}_{3}$ film at temperatures of $480^{\circ} \mathrm{C}$ and $490^{\circ} \mathrm{C}$. It can be seen from these dependences that the change in the temperature in a narrow temperature range near the Curie point does not significantly affect the threshold of switching cycles but significantly affect the magnitude of switchable polarization.

Method of experimental investigations of fatigue on the Sawyer-Tower circuit allows to determine the number of switching cycles, when a critical decline in the switching polarization arises, but it does not reveal which fatigue mechanism to prefer. So, together with Sawyer-Tower method in this paper we used the registration of a switching current under the influence of rectangular field pulses by the method of Mertz. Such measurements make it possible not only to register the resulting change of the polarization, but also to trace the changes that occur in separate stages of the switching in the fatigue process

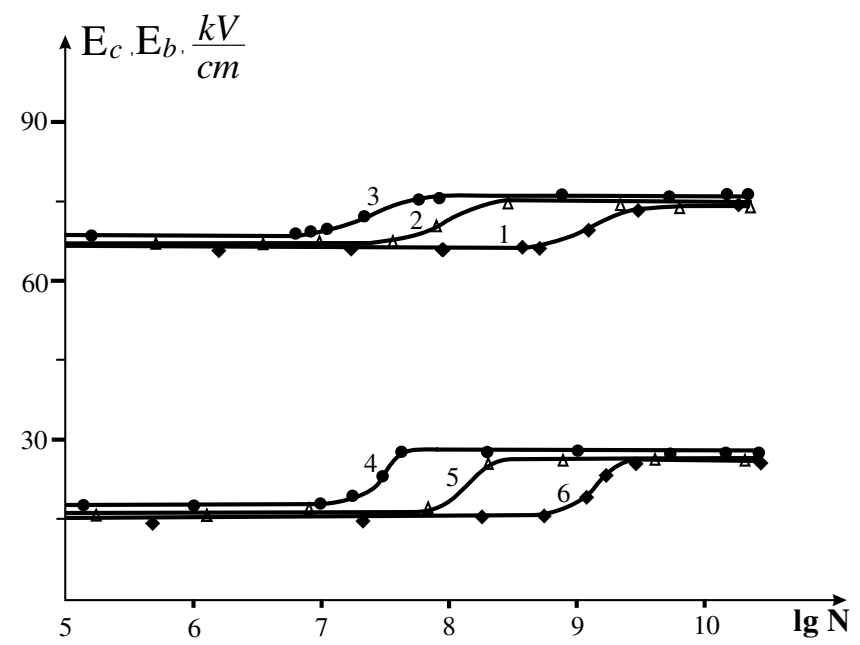

Fig. 6. Dependencies of coercive field (curves 1,2,3) and internal bias field (curves 4,5,6) on logarithm of the number of switching cycles for $\mathrm{PbTiO}_{3}$ films on silicon substrate. $T=200^{\circ} \mathrm{C}$, $f=10 \mathrm{MHz}: 1,6 ; 2,5 ; 3,4-E=130,200,270 \mathrm{kV} / \mathrm{cm}$.

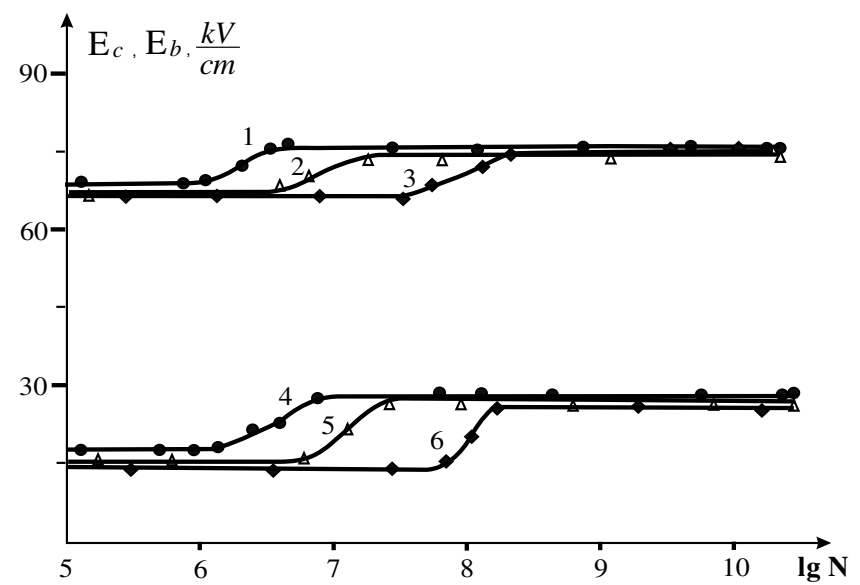

Fig. 7. Dependencies of coercive field (curves 1,2,3) and internal bias field (curves 4,5,6) on logarithm of the number of switching cycles for $\mathrm{Pb}\left(\mathrm{Zr}_{0.5} \mathrm{Ti}_{0.5}\right) \mathrm{O}_{3}$ films on silicon substrate. $T=200^{\circ} \mathrm{C}$, $E=130 \mathrm{kV} / \mathrm{cm}: 1,4-100 \mathrm{kHz} ; 2,5-1 \mathrm{MHz} ; 3,6-10 \mathrm{MHz}$.

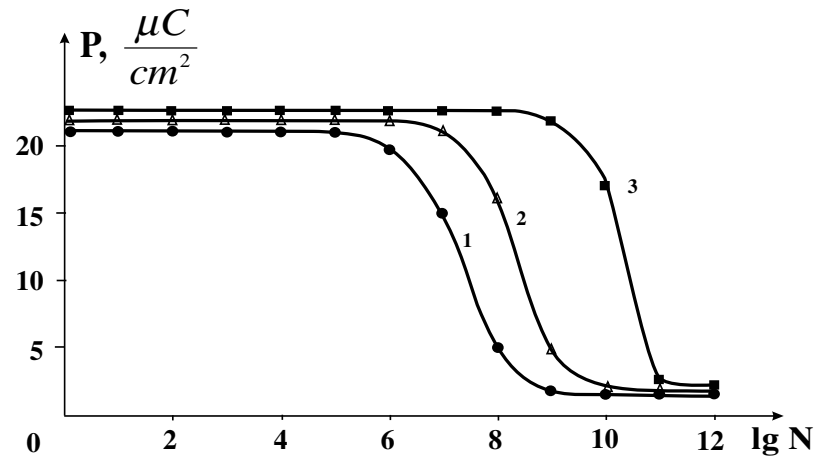

Fig. 8. Dependencies of the switched polarization on the number of switching cycles for $\mathrm{PbTiO}_{3}$ films on silicon substrate at $T=200^{\circ} \mathrm{C}, E=130 \mathrm{kV} / \mathrm{cm}$ in sinusoidal fields: $1-100 \mathrm{kHz} ; 2-$ $1 \mathrm{MHz} ; 3-10 \mathrm{MHz}$.

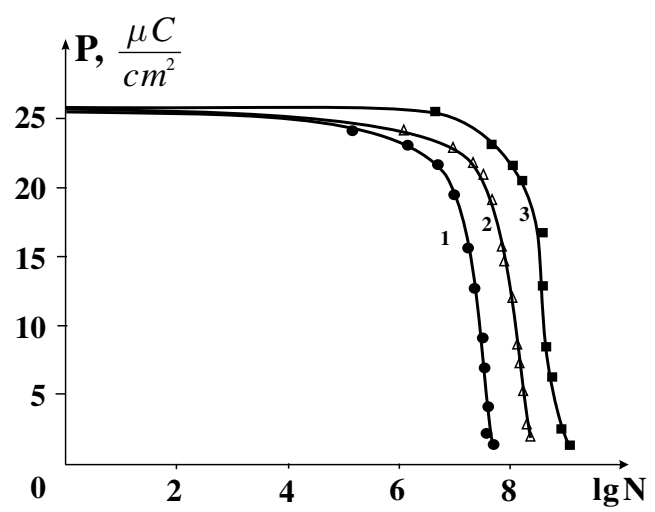

Fig. 9. Dependencies of the switched polarization on the number of switching cycles for $\mathrm{Pb}\left(\mathrm{Zr}_{0.5} \mathrm{Ti}_{0.5}\right) \mathrm{O}_{3}$ films on silicon substrate at $T=200^{\circ} \mathrm{C}, E=130 \mathrm{kV} / \mathrm{cm}: 1-100 \mathrm{kHz} ; 2-1 \mathrm{MHz} ; 3-$ $10 \mathrm{MHz}$. 


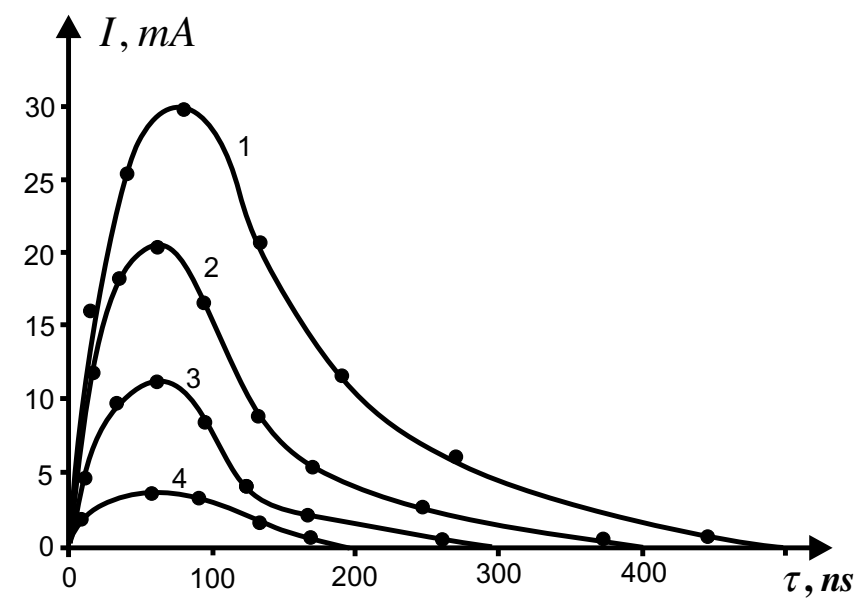

Fig. 10. Time evolution of positive pulses of the switching current for different numbers of switching cycles $N$ for lead titanate films with thickness of $200 \mathrm{~nm}$ with an external field of $200 \mathrm{kV} / \mathrm{cm}$ : $N=1-10^{6}, 2-10^{7}, 3-10^{8}, 4-10^{9}$.

Time dependences of the switching current for the films of lead titanate after different numbers of switching cycles are shown in Fig. 10. With increasing the number of switching cycles $N$, a decrease of the maximum current and the switching time were observed. Using the obtained switching current $i$ dependencies we can calculate the switching charge

$$
Q=\int i(t) d t=2 P S,
$$

( $S$ is the area of electrodes), the switchable polarization $P$ and obtain its dependence on the number of switching cycles $N$.

As expected, thus constructed dependences $P(N)$ with the varying values of the external field, frequencies, and thicknesses of the ferroelectric films confirms the information obtained from the analysis of the changes in the hysteresis loop. Namely, when the voltage increases, the switchable polarization drop occurs at the lower number of cycles $N$, the threshold of switching cycles withstood by the film increases with increasing frequency.

It was found, that processes of repolarization fatigue in the films of lead titanate and lead zirconate titanate are more intensive under the rectangular pulses, than for sinusoidal shape signal at equal frequency and signal amplitude (Fig. 11).

To detect possible changes of switching kinetics in the fatigue process of thin films we constructed dependences of the maximum switching current $i_{\max }$ on the applied field $E$ for the samples with different numbers of switching cycles in the fields above the coercive (Fig. 12). As can be seen, these dependences are linear, which is typical for the case of viscous motion of domain walls:

$$
i_{\max }=\operatorname{const}\left(E-E_{b}\right),
$$

where $E$ - external field, $E_{b}$ - internal bias field.

Note that experimental dependencies at the Fig. 12 are almost parallel to each other. It means that the mobility of

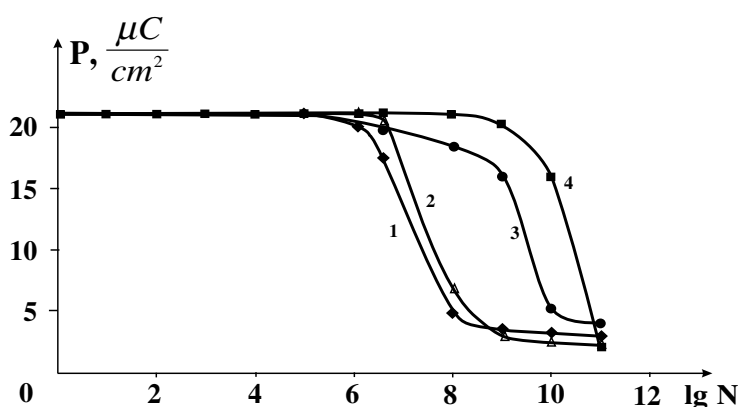

Fig. 11. Comparison of changes in polarization on the number of switching cycles for $\mathrm{PbTiO}_{3}$ films with thickness of $200 \mathrm{~nm}$ at rectangular $(1,3)$ and sinusoidal $(2,4)$ external field. 1,2 $E=250 \mathrm{kV} / \mathrm{cm}, 3,4-E=160 \mathrm{kV} / \mathrm{cm}$.

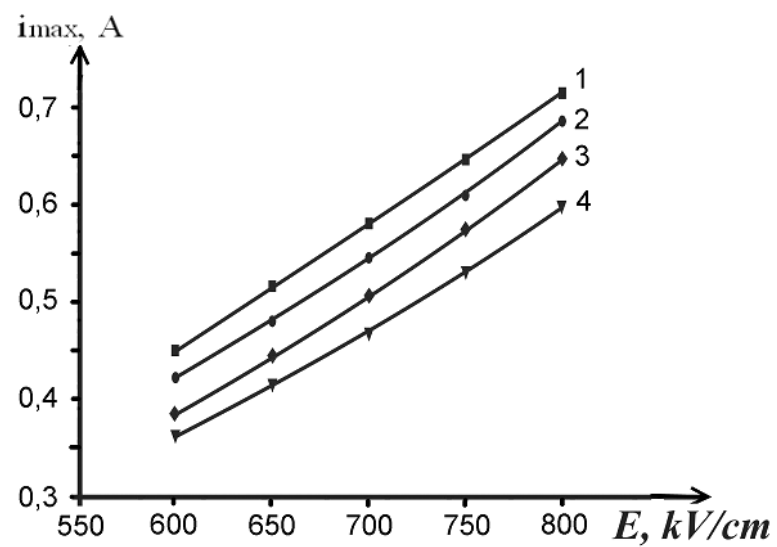

Fig. 12. The dependence of $i_{\max }$ on the field for the films of lead zirconate titanate with thickness of $100 \mathrm{~nm}$ after different number of switching cycles at a frequency of $10 \mathrm{kHz}: N=1-10^{6}, 2-10^{7}$, $3-10^{8}, 4-10^{9}$.

domain walls, which determines the coefficient of proportionality in the last expression, is unchanged when the number of switching cycles increases. Therefore, fatigue processes, at least, are not related with the changes in the mobility of domain walls.

\section{Discussion of the Results}

Speaking about the nature of the observed fatigue processes, we note the following. The absence of change in the phase composition of the films exposed to the phenomenon of fatigue, indicates the nonchemical nature of the observed phenomenon. It is known ${ }^{20}$ that the changes arising in the material as a result of fatigue phenomena partly restored after 1.5 months. In addition, no mechanical damage is observed on the surface of the "tired" samples (the electrodes destruction, etc.). This indicates that observed processes of fatigue obviously do not represent mechanical damages, which cannot be easily recoverable. 


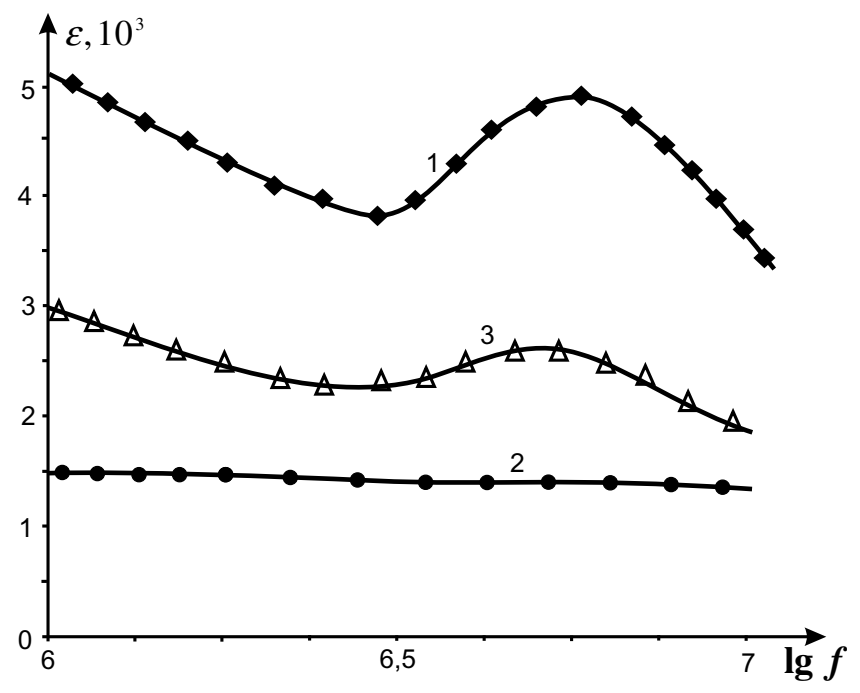

Fig. 13. The frequency dependencies of maximum value of real part of dielectric constant for $\mathrm{PbTiO}_{3}$ film: 1 - initial measurement, 2 after $10^{11}$ switching cycles, 3 - after recovery period of 1.5 months. $T=495^{\circ} \mathrm{C}$.

The studies of the frequency dependence of the dielectric permittivity in the fatigue process and the comparison of obtained results with the primary measurements data showed that in particular for the samples of lead titanate synthesized by magnetron sputtering, in the vicinity of $14 \mathrm{MHz}$ the primary measurements exhibit first a decrease, and then a hump (Fig. 13) that disappeared at temperatures above the phase transition point. The corresponding frequency matches the natural vibration frequency of domain walls calculated theoretically and approximately equal to $10^{7} \mathrm{~Hz}^{20}$ The absence of this anomaly in the paraelectric phase and the coincidence of the frequency range in which this anomaly is experimentally observed with theoretical estimates allow to suggest a domain origin of the observed anomaly in the experiment.

After $10^{11}$ switching cycles the above mentioned $\varepsilon^{\prime}(f)$ anomaly at $\sim 10^{7} \mathrm{~Hz}$ disappears (Fig. 13) and is then partially restored after 1.5 months (Fig. 13, curve 3). The sensitivity of the dielectric anomaly at frequencies about $10^{7} \mathrm{~Hz}$ having a domain origin to the phenomenon of fatigue clearly indicates the domain-related nature of the fatigue itself.

As noted, we used the electric fields larger than coercive to stimulate the switching of polarization. Obviously, any field with an amplitude, greater than this value will lead to repolarization and, consequently, further increase the amplitude of the field above the coercive does not matter in terms of the switching and related processes. The presence of the actually observed dependence of fatigue on the field amplitude in this area indicates on other nature of fatigue processes which is not related with the actual switching process. Such a process, clearly, can be the charge drift in the applied electric field, where the growth of its amplitude should accelerate the motion of charge carriers. The presence of the

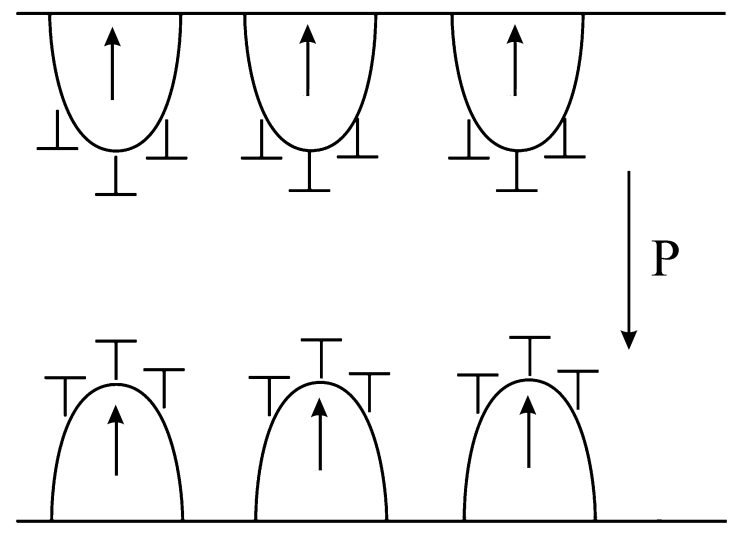

Fig. 14. Fixing of reverse domains by lattice dislocations. Vectors are indicated the directions of spontaneous polarization vectors in the bulk of the crystal and in the inverse domains.

depolarizing field near the ferroelectric surface forms the system of inverse domains here, which are not stable and should disappear with time in the absence of the screening of bound charges and the compensation for elastic stresses in the region of extremities of these domains. Redistribution of the spatial distribution of charge carriers in electric field can fix the system of inverse domains arising near the ferroelectric surface.

The role of pinning defects in the crystal structure can be played by charged point defects and dislocations, which compensate the bound charge and the elastic stresses at the end of the inverse domains. The obvious problem is the type of compensating carriers. Conventional charged-point carriers, obviously, do not give a contribution to this effect, because the symmetrical variation of the alternating field does not create a preferential direction of acting force, which is necessary for the directed motion of charged carriers. At the same time, the role of these carriers can be played by dislocations. Dislocations in ferroelectric crystals are charged and therefore are exposed to the displacing force of applied field. The sign of the dislocation charge varies with the change of spontaneous polarization vector, so a given dislocation in the same external field will experience forces of opposite sign in different domains. When the orientation of the polarization vector changes, direction of the force acting on a dislocation by the external field changes too. As a result, the time-averaged force acting on charged dislocation during a switching cycle, is not equal to zero (Fig. 15). In other words, there is a constant component of the force, driving the dislocations in the same direction during the periodic switching of the ferroelectric sample. Long-term effects of such a force leads to the separation of the opposite sign dislocations, ${ }^{23}$ which leads to the accumulation of necessary elastic and compensative electric fields near the surface of the film or at the extremities of inverse domains.

The critical value of the number of switching cycles can be estimated from the condition of the equality of the switching polarization (i.e., the surface charge density on the 


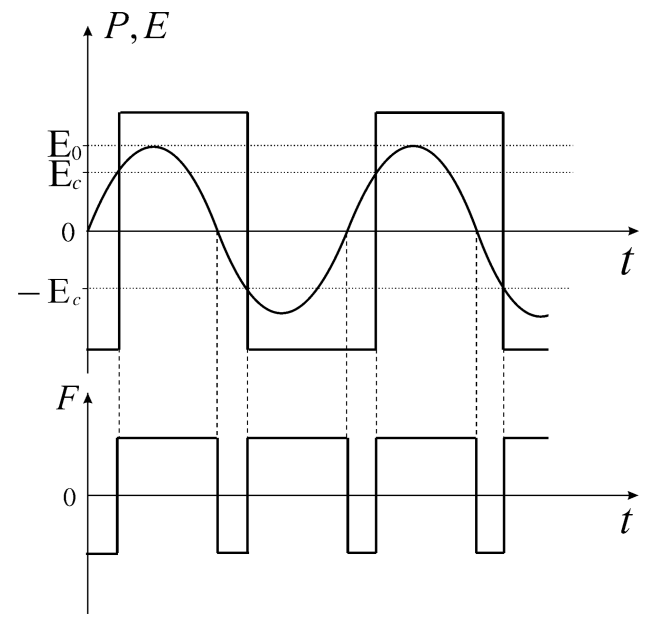

Fig. 15. The appearance of nonzero average force acting on the charged dislocations during cyclic switching of a ferroelectric: 1 the applied sinusoidal field, 2 - change polarization, 3 - a sign of the force acting on a charged dislocation as a function of switching time.

ferroelectrics surface) to the charge density carried here by a stream of charged dislocations for a time equal to the total duration of the critical number of switching cycles:

$$
P=\Delta P \cdot N=N \int j d t .
$$

In (3) $P$ is switching polarization, $N$ critical number of the switching cycles, and the integration is over one switching cycle.

The surface current density $j$ is associated with the motion of charged dislocations; it may be written as:

$$
j=\tau n_{s} \mu E,
$$

where $\tau=P b-$ linear charge density of dislocation, ${ }^{24} b-$ Burgers vector of dislocation, $n_{s}$ - the surface density of dislocations, $\mu-$ the mobility of dislocations, $E-$ the electrical field acting on a unit of dislocation length.

To limit the rectangular hysteresis loop (Fig. 16) the influence on charged dislocations at sites $\mathrm{BC}$ and FD are

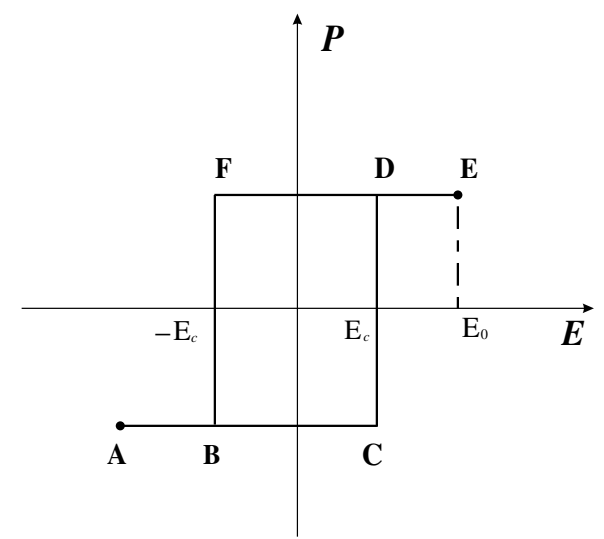

Fig. 16. A rectangular dielectric hysteresis loop. canceling each other out. Uncompensated influence is realized only on the "whiskers" hysteresis loop — sites AB and DE.

We assume now that the switching of the ferroelectric film is implemented by a harmonic signal

$$
E=E_{0} \sin 2 \pi f t,
$$

with $E_{0}$ as amplitude and $f$ as switching frequency.

For an undisplaced hysteresis loop (in the absence of internal bias field) $\mathrm{AB}$ equal to $\mathrm{DE}$. In this case, the charge transferred by the current with density $j$ through a unit surface area is

$$
\Delta P=4 \int_{t_{1}}^{t_{2}} j d t
$$

where times $t_{1}$ and $t_{2}$ are determined by the conditions $E=$ $E_{c}$ and $E=E_{0}$, respectively, and are equal to:

$$
t_{1}=\frac{1}{2 \pi f} \arcsin \left(E_{c} / E_{0}\right), \quad t_{2}=\frac{1}{4 f} .
$$

Calculating $\Delta P$ (6) subject to (7), on the basis of ratio (3) we obtain

$$
N=\frac{f}{4 n_{s} \mu b \sqrt{E_{0}^{2}-E_{c}^{2}}} .
$$

As seen from (8), all the experimentally observed dependencies of the fatigue on the key parameters of the switching process (such as field amplitude, frequency and temperature) may be explained by the proposed model.

In particular, the critical number of switching cycles decreases with increasing length of the "whiskers", i.e., difference between $E_{0}$ and $E_{c}$. Herewith, at the same value of whiskers $E_{0}-E_{c}$ when the switching process is carried out in the region of strong fields due to increasing the difference of squares $E_{0}^{2}-E_{c}^{2}=\left(E_{0}-E_{c}\right)\left(E_{0}+E_{c}\right)$ switching in a stronger field causes more intense drift of charged carriers and, therefore, more intensive processes of fatigue. This may explain in particular the experimentally observed increase in the fatigue in samples with a greater magnitude of the coercive field. ${ }^{25}$

The critical number of switching cycles decreases with growth of the temperature and with decreasing frequency also. Reducing $N$ with increasing temperature in this model is explained by the increased mobility of dislocations $\mu$. The reason for the reduction of the "fatigue" processes (increasing the number of cycles $N$, which the sample can withstand) with the increase of the frequency in present case is not connected with any peculiarities of high frequency motion of the domain wall itself, but is stipulated by the decreasing of one cycle duration of action of the electric field, promoting the movement of charged dislocations. In other words, resultant state of fatigue is determined by general time spent by the sample on areas corresponding to "whiskers" of a hysteresis loop. Herewith, a key role of the switching in this case is to change the sign of charge on the dislocation 
that provides unidirectional exposure and, therefore, unidirectional movement of the same dislocation on hysteresis loop "whiskers" with different polarity in an alternating field due to simultaneous changes here in the sign of the field and the sign of a charge on a dislocation. While for point charged defects with invariable sign of charge such effects on loop's "whiskers" with different polarity will cancel each other.

According to Fig. 12, with growing number of switching cycles the point of intersection of the dependence of maximum switching current on the field with the field axis is shifted to the right, which means a corresponding increase in the internal bias field.

The role of the generalized factor called internal biasing field, as we know, can play defects conducive to the creation a preferred direction of polarization of the sample, for example, dislocations of the same sign arising in the surface layer due to different coefficients of thermal expansion of the ferroelectric film and the substrate. ${ }^{26}$ In this case, experimentally observed increase of the internal bias field in the fatigue process with growth of the switching cycles number can be explained by a gradual increase with time of the charged dislocations number near the film surface, pinning here the polarization of a certain direction.

The experimental results on the research of impact of the signal forms to processes of the repolarization fatigue in the films of lead titanate and lead zirconate titanate can be explained by the difference in time of an action to a charge carrier of the higher field value. For rectangular pulses the time of exposure on the film is larger than for sinusoidal fields at the same frequency and same external field amplitude, which explains the high intensity of the fatigue of switching ferroelectric materials in the fields of rectangular shape.

\section{Conclusion}

The research of the fatigue processes of thin ferroelectric films of lead titanate $\mathrm{PbTiO}_{3}$ and lead zirconate titanate $\mathrm{Pb}(\mathrm{Zr}, \mathrm{Ti}) \mathrm{O}_{3}$ using the Sawyer-Tower circuit and the method of Mertz allowed to express quite reasonable assumption about the nature of the observed phenomena and to explain its patterns in the framework of the diffusion of charged carriers to the film surface, leading to fixation of the polarization and thus — blocking the switching processes. The efficiency of this process increases with increasing the field value, exposure time and temperature, which explains the experimentally observed dependencies.

The defects, that lead to the accumulation of fatigue, related with polarization switching, are charged dislocations. They are changing sign with the change in the polarity of their domain, and are having impact by permanent forces in a certain direction, in contrast to charged point defect. In this model, the numerical estimates allow to obtain reasonable values of the critical number of switching cycles and the relaxation time corresponding to the experiment.

\section{References}

${ }^{1}$ S. V. Kalinin, A. Gruverman, B. J. Rodriguez, J. Shin, A. P. Baddorf, E. Karapetian and M. Kachanov, J. Appl. Phys. 97, 074305 (2005).

${ }^{2}$ A. Gruverman and S. V. Kalinin, J. Mater. Sci. 41, 107 (2006).

${ }^{3}$ A. Roelofs, T. Schneller, K. Szot and R. Waser, Appl. Phys. Lett. 81, 5231 (2002).

${ }^{4}$ S.-J. Kim and Q. Jiang, Smart Mater. Struct. 5, 321 (1996).

${ }^{5}$ E. L. Colla, A. K. Tagantsev, D. Taylor and A. L. Kholkin, J. Korean Phys. Soc. (Proc. Suppl.) 32, 1353 (1998).

${ }^{6}$ D. C. Lupascu, E. Aulbach and R. Jurgen, J. Appl. Phys. 93, 5551 (2003).

${ }^{7}$ A. S. Sidorkin, Proc. Ninth IEEE Int. Symp. Applications of Ferroelectrics (1994), p. 91.

${ }^{8}$ A. S. Sidorkin, Domain Structure in Ferroelectrics and Related Materials (Cambridge International Science Publishing, United Kingdom, 2006).

${ }^{9}$ W. L. Warren, D. Dimos, B. A. Tuttle, G. E. Pike, R. W. Schwartz, P. J. Clew and D. C. McIntyre, J. Appl. Phys. 77, 6695 (1995).

${ }^{10}$ E. L. Colla, A. K. Tagantsev, D. V. Taylor and A. L. Kholkin, Integr. Ferroelectr. 18, 19 (1997).

${ }^{11}$ A. K. Tagantsev and I. A. Stolichnov, Appl. Phys. Lett. 74, 1326 (1999).

${ }^{12}$ I. Stolichnov, A. K. Tagantsev, E. Colla, S. Gentil, S. Hiboux, J. Baborowski, P. Muralt and N. Setter, J. Appl. Phys. 88, 2154 (2000).

${ }^{13}$ X. Du and I.-W. Chen, J. Appl. Phys. 83, 7789 (1998).

${ }^{14}$ I. K. Yoo and S. B. Desu, Phys. Stat. Sol. A 133, 565 (1992).

${ }^{15}$ M. Dawber and J. F. Scott, Appl. Phys. Lett. 76, 1060 (2000).

${ }^{16}$ P. K. Larsen, G. J. M. Dormans, D. J. Taylor and P. J. Van Veldhoven, J. Appl. Phys. 76, 2405 (1994).

${ }^{17}$ E. Paton, M. Brazier, S. Mansour and A. Bement, Integr. Ferroelectr. 18, 29 (1997).

${ }^{18}$ C. Pawlaczyk, A. K. Tagantsev, K. Brooks, I. M. Reaney, R. Klissurska and N. Setter, Integr. Ferroelectr. 9, 293 (1995).

${ }^{19}$ A. S. Sidorkin, L. P. Nesterenko, A. L. Smirnov, G. L. Smirnov, S. V. Ryabtsev and A. A. Sidorkin, Phys. Solid State 50, 2157 (2008).

${ }^{20}$ A. Sidorkin, L. Nesterenko, A. Sidorkin, S. Ryabtsev and G. Bulavina, Solid State Sci. 12, 302 (2010).

${ }^{21}$ A. S. Sidorkin, L. P. Nesterenko, A. L. Smirnov, G. L. Smirnov and S. V. Ryabtsev, Ferroelectrics 349, 171 (2007).

${ }^{22}$ A. S. Sidorkin, L. P. Nesterenko, G. L. Smirnov, A. L. Smirnov and S. V. Ryabtsev, Phys. Solid State 48, 1189 (2006).

${ }^{23}$ A. S. Sidorkin, L. P. Nesterenko, B. M. Darinskii, A. A. Sidorkin and G. G. Bulavina, Crystallogr. Rep. 56, 35 (2011).

${ }^{24}$ S. P. Alpay, I. B. Misirlioglu, V. Nagarajan and R. Rames, Appl. Phys. Lett. 85, 2044 (2004).

${ }^{25}$ A. S. Sidorkin, L. P. Nesterenko, B. M. Darinskii, A. A. Sidorkin and E. V. Ionova, Bull. Russian Acad. Sci.: Phys. 74, 1311 (2010).

${ }^{26}$ S. Y. Hu, Y. L. Li and L. Q. Chen, J. Appl. Phys. 94, 2542 (2003). 